\title{
Generation Laser Scanning Method for the Visualization of Ultrasounds Propagating on a 3-D Object with an Arbitrary Shape
}

\author{
Junji TAKATSUBO $^{* *}$, Bo WANG ${ }^{* *}$, Hiroshi TSUDA ${ }^{* *}$ and Nobuyuki TOYAMA ${ }^{* *}$ \\ ${ }^{* *}$ Research Institute of Instrumentation Frontier, AIST, \\ Tsukuba Central2, Tsukuba Umezono 1-1-1, 305-8568, Japan \\ E-mail: no@input
}

\begin{abstract}
We propose a generation laser scanning method for imaging of the propagation of ultrasounds. By scanning a pulsed-laser beam on an object for the generation of ultrasounds, the propagated ultrasounds were detected by a fixed PZT transducer. Although the detected waves were ultrasounds generated from different irradiation points, we could, by means of simple data processing, produce the dynamic images of the ultrasounds generated from the reception transducer position. Since this method requires no adjustment of the beam incident angle or beam focus, we can easily visualize the ultrasound propagating on a solid media having a complicated shape. The distinguishing feature of this method is its applicability in the field of inspection work.
\end{abstract}

Key words: Ultrasound, Non-Destructive Inspection, Visualization, Laser, Defect

\section{Introduction}

In the field of ultrasonic flaw detection, the inspection of defects by the observation and analysis of signal waveforms is common. However, the propagation characteristics of ultrasonic waves vary depending on the type and coupling state, as well as on the mounting position of the sensor being used and the material and shape of the object being inspected. In areas having complex shapes, reflected waves, diffracted waves, and mode-converted waves propagate while interfering with each other, making it difficult for even an expert inspector to accurately detect defect echoes within a short time. If it were possible to perform inspections while observing the propagation behavior of ultrasonic waves as images, the identification of defect echoes would become easier, and quicker inspection would become possible. Furthermore, fewer defects would be overlooked or improperly identified, leading to higher-reliability inspections.

The Schlieren method ${ }^{(1)-(3)}$ and the photo-elasticity method ${ }^{(4),(5)}$ are known methods for visualizing the propagation of ultrasonic waves. However, both of these methods are used to visualize ultrasonic waves propagating in transparent media and cannot be applied to opaque objects. Although visualization by numerical analysis with computer simulation has been implemented, ${ }^{(6)-(9)}$ the necessary calculations are time consuming, even with the latest computers, so only objects with simple forms can be analyzed with high accuracy. Furthermore, even if ultrasonic waves propagating on an object with a complex form could be visualized using a high-performance computer, it is impossible to detect defects by simulation.

The detection laser-probe scanning method ${ }^{(10)}$, which we proposed in a previous study,

*Received 19 Sep., 2007 (No. T1-05-1075) Japanese Original : Trans. Jpn. Soc. Mech. Eng., Vol.72, No.718, A (2006), pp.945-950 (Received 7 Oct., 2005) [DOI: 10.1299/jmmp.1.1405] 
enables the visualization of ultrasonic waves propagating on the surface of an opaque object. With this method, however, it is necessary to apply laser beams perpendicularly to the specimen and to keep the focal distance constant while scanning the object, making it difficult to visualize ultrasonic waves propagating on a 3-D object of arbitrary shape. We have recently developed a method for visualizing the propagation of ultrasonic waves by scanning generation pulsed-laser rather than detection laser-probe. The proposed method has the following features, which make it superior to the conventional detection-probe scanning method. (1) The proposed method enables the visualization of ultrasonic waves propagating on a complex-shaped object with curved surfaces, steps, and dents. (2) The proposed method provides excellent working efficiency by eliminating the need for adjustments to the laser incidence angle or the focal distance. (3) The proposed method has high detection sensitivity. (4) The proposed method enables remote measurement of images of ultrasonic waves propagating over a wide area. For these reasons, we believe that the proposed method can be effectively applied to the inspection of defects in the field. In the present paper, we provide an outline of the proposed visualization method and present examples of visualization.

\section{Principle of Visualization}

When ultrasonic waves are detected propagating between two points using a pair of transmission-reception transducers having the same characteristics, the same waveform should be detected if the transmission-reception transducers were switched. Similarly, the waves detected by the PZT (piezoelectric) sensor at point B from radiating laser beams at point $\mathrm{A}$ would be almost the same as the waves detected at point $\mathrm{A}$ from radiating laser beams at point $\mathrm{B}$. The principle of visualization is based on this reciprocity of ultrasonic propagations. To confirm this reciprocity, even when there is a defect in the propagation path of the ultrasonic waves, a slit crack with a width of $1 \mathrm{~mm}$ and a length of $35 \mathrm{~mm}$ was created in an aluminum specimen, as illustrated in Fig. 1. We then checked whether the ultrasonic waves detected by the PZT sensor (outer diameter: 3 $\mathrm{mm})$ at point $A$ with laser beams radiated from point $B$ were equal to the waves detected by the PZT sensor at point B with laser beams radiated from point A. As shown in Fig. 2, the two waveforms are approximately the same. The lack of complete agreement between the two waves arises because it is difficult to mount the PZT sensors with good reproducibility. In addition, the reciprocity of ultrasonic propagation is not necessarily applied in this measurement because the spot diameter of the

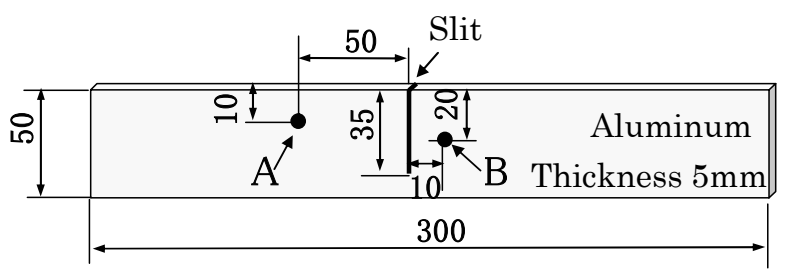

Fig.1 Aluminum specimen with a slit.

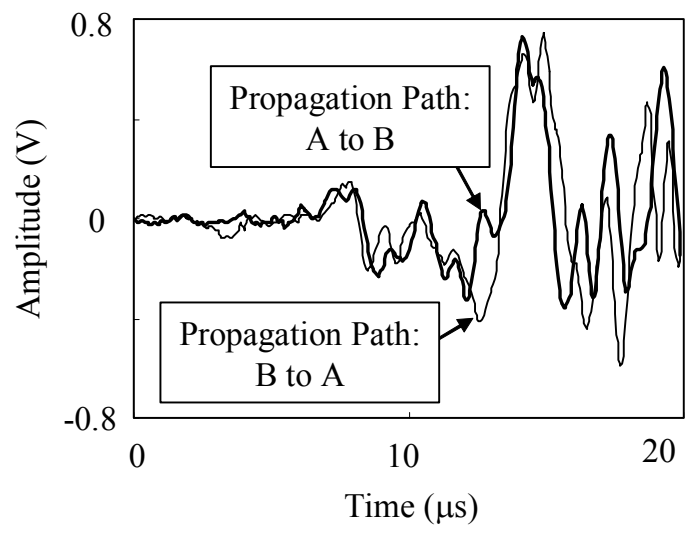

Fig.2 Comparison of the detected waveforms when the propagation path between the laser irradiation point and the reception sensor position is reversed. 
laser beam (approximately $2 \mathrm{~mm}$ ) differs from the diameter of the receiving section of the piezoelectric sensor $(3 \mathrm{~mm})$. The observed waveform is a Lamb wave with a velocity of approximately $2,800 \mathrm{~m} / \mathrm{s}$. In contrast, the observed wave bundle near $8 \mu$ s consists of diffraction waves from the top of the slit, and the wave bundle near $14 \mu$ s consists of the reflected waves from the bottom side.

If it is assumed that the propagation of ultrasonic waves is reciprocal, the train of waveforms detected by the PZT sensor at a fixed position with the scanning of a generation laser will be the same as the train of waveforms detected by the scanning of the reception PZT sensor with the irradiating laser beam at the fixed position. Therefore, dynamic images of ultrasonic propagation from the fixed point may be created by making a time series contour map of the displacement of the detected waves.

\section{Visualization System}

An outline of the visualization system is shown in Fig. 3. Pulsed-laser beams (YAG, pulse width: $10 \mathrm{~ns}$, beam diameter: $2 \mathrm{~mm}$, energy: $5 \mathrm{~mJ}$ ) are irradiated onto the specimen at a frequency of $20 \mathrm{~Hz}$. The pulsed-laser is placed on a double-axle rotating stage, and the irradiation point of the laser beams is scanned in the form of a grid by controlling the rotation angle of the stage with a PC. While scanning the measurement points arranged on a $100 \times 100$

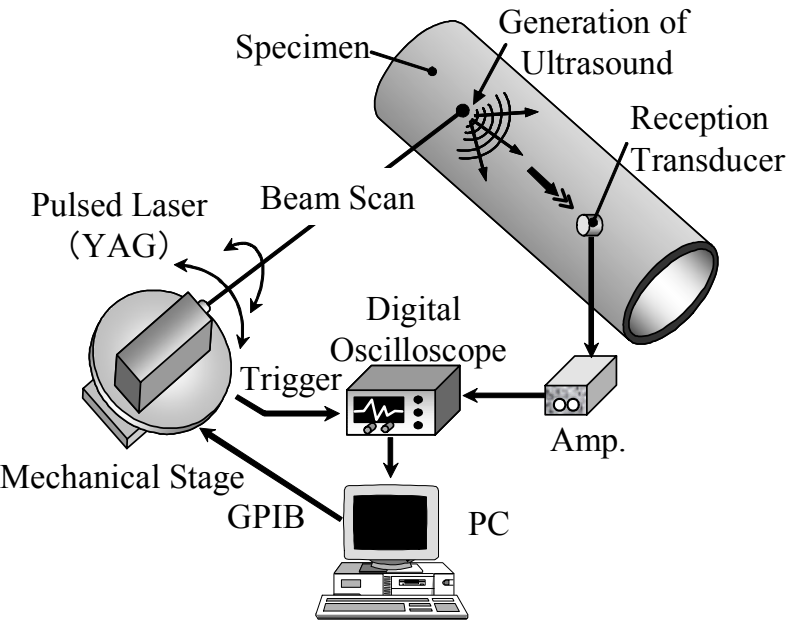

Fig.3 Pulsed laser system for visualizing ultrasonic waves. grid for a total of 10,000 points, thermally excited ultrasonic waves generated at each irradiation point are detected by the reception PZT sensor and are recorded on the PC via an amplifier and a digital oscilloscope (A/D converter). If the reciprocity of ultrasonic propagation is applied, the amplitudes of these trains of waveforms at a given point in time correspond to the displacement of the ultrasound propagated from the reception sensor. The ultrasonic propagation can therefore be observed when the displacement contour maps are displayed consecutively. Although these images, being based on actual measurement data, do not represent ultrasonic waves that are actually propagating, they are images of ultrasonic waves that can actually exist. The propagation time for each visualized image represents a relative time and, for convenience, is initially set to $0 \mathrm{sec}$. The laser scanning and recording of the waveforms is performed at a rate of approximately $10 \mathrm{~Hz}$, and so it takes approximately $15 \mathrm{~min}$ to record the waveforms for $100 \times 100$ points. The received waveforms were not averaged.

\section{Verification of Appropriateness of the Proposed Method for Visualizing the Propagation of Ultrasonic Waves}

To verify whether the images of the propagation of ultrasonic waves obtained by the proposed method are appropriate, we compared the images obtained using the proposed with images obtained using the conventional method of scanning the detection laser. A 40 
$\mathrm{mm} \times 40 \mathrm{~mm}$ piece of aluminum alloy (thickness: $11 \mathrm{~mm}$ ) in which a copper rod having a diameter of $10 \mathrm{~mm}$ was embedded, as illustrated in Fig. 4, was used as a specimen.

When images were created using the conventional method, spike waves were transmitted periodically from an ultrasonic pulse generator to the reception sensor (outer diameter: $20 \mathrm{~mm}$, nominal frequency: $2 \mathrm{MHz}$ ) mounted on the side of the specimen to generate ultrasonic waves. The visualized images were obtained by measuring the displacements of the

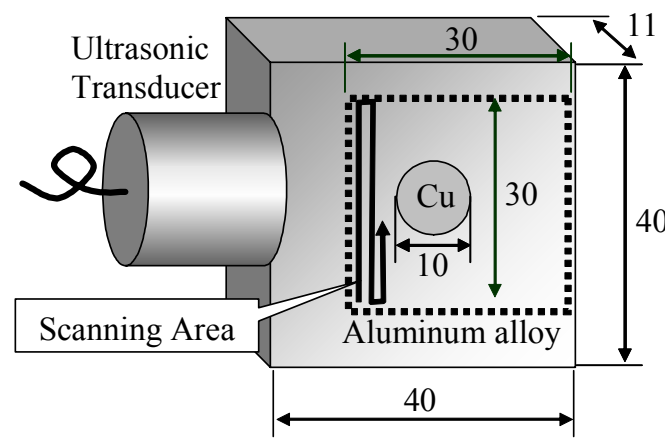

Fig.4 Experimental setup for the visualization of ultrasonic waves propagating around a copper rod embedded in an aluminum alloy. ultrasonic waves propagating on the surface of the specimen using a detection laser probe.$^{(10),(11)}$ The detection laser probe scanned a $30 \mathrm{~mm} \times 30 \mathrm{~mm}$ area of the specimen at intervals of $0.3 \mathrm{~mm}$. Using the proposed method, pulsed-laser beams scanned the above-described area and generated thermal excited ultrasounds. The propagation images are created based on the waves detected by the reception sensor mounted on the side of the specimen. Figure 5 compares the results obtained using the respective methods. The images of reflection, diffraction, and transmission around the copper rod are almost the same for both methods, which demonstrates the appropriateness of the proposed method.
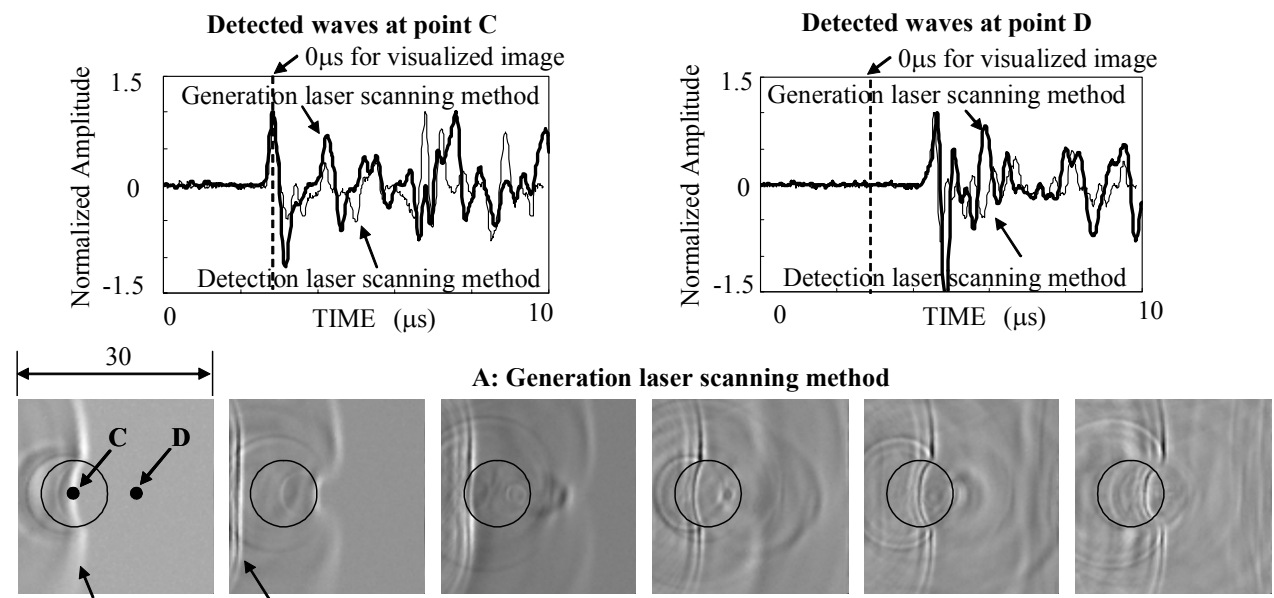

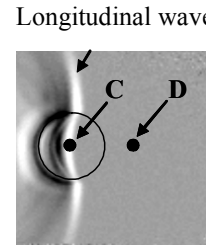

$0 \mu \mathrm{s}$

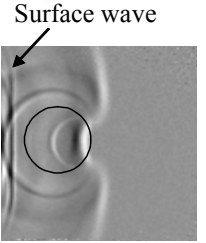

$1 \mu \mathrm{s}$

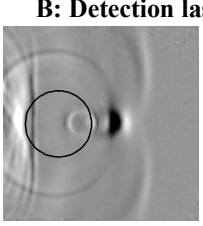

$2 \mu \mathrm{s}$

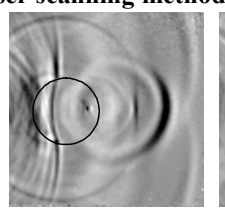

$3 \mu \mathrm{s}$

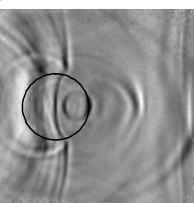

$4 \mu \mathrm{s}$

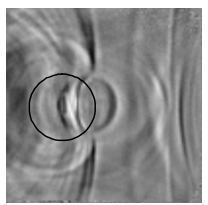

$5 \mu \mathrm{s}$

Fig.5 Visualization of ultrasonic waves propagating around a copper rod embedded in an aluminum alloy.

\section{Examples of Visualized Propagation of Ultrasonic Waves}

Next, we introduce a sample image of ultrasonic waves propagating on a drill surface as an example of the visualization of ultrasonic waves propagating on a 3-D object with a curved surface. As shown in Fig. 6, a PZT sensor (sensitivity diameter: $4 \mathrm{~mm}$, nominal resonant frequency: $300 \mathrm{kHz}$ ) is mounted on the surface of the drill, and a $100 \mathrm{~mm} \times 50 \mathrm{~mm}$ 


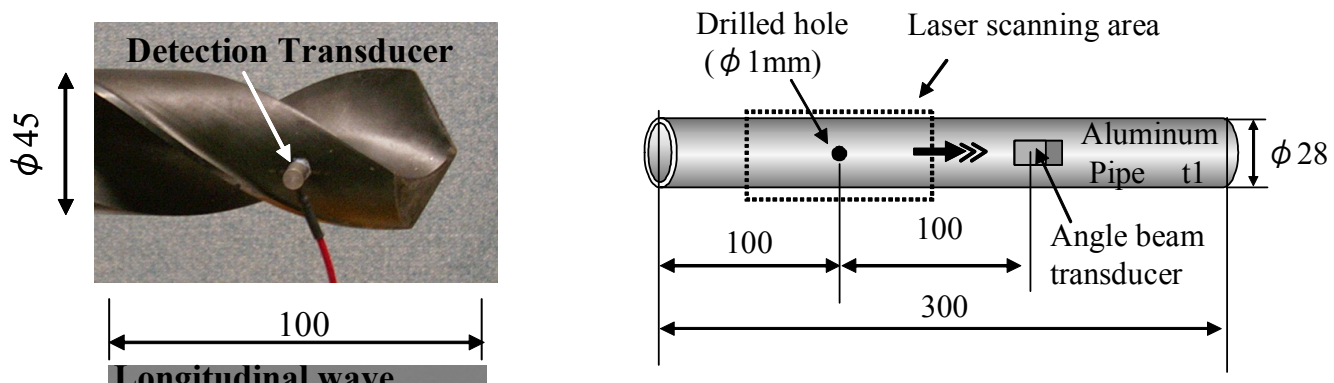

$0 \mu \mathrm{s}$

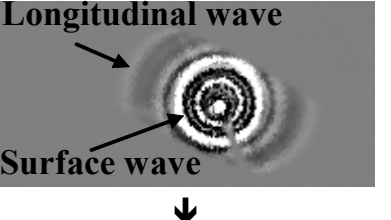

$5 \mu \mathrm{s}$

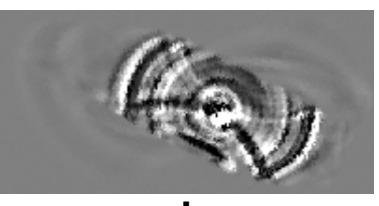

$\downarrow$

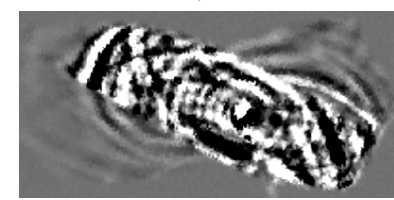

$\downarrow$

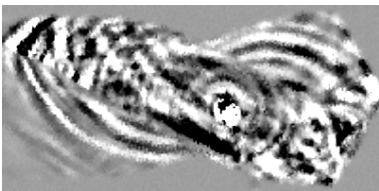

$15 \mu \mathrm{s}$

Fig.6 Visualization of an ultrasonic wave propagating on a drilled surface.

area was scanned by laser at intervals of approximately $0.5 \mathrm{~mm}$ (beam rotation pitch: $0.025^{\circ}$ ) to obtain images of the propagation of ultrasonic waves. The laser beam is approximately $1 \mathrm{~m}$ in length, and the sampling interval of the detected waveforms is $0.2 \mu \mathrm{s}$. The number of sampling points is 500. Figure 6 presents visualized images for each $5 \mu$ s of propagation time. Although computer simulation of ultrasonic waves propagating on an object of complex-spiral form would seem difficult, visualization may be accomplished within a short time using the proposed method.

We also tried to visualize ultrasonic waves propagating on an aluminum tube with the aim of applying this method to detect flaws in pipes. As shown in Fig. 7, a drill hole is opened at a position $100 \mathrm{~mm}$ from the left end of the aluminum tube with a length of 300 $\mathrm{mm}$, an outer diameter of $28 \mathrm{~mm}$, and a thickness of $1 \mathrm{~mm}$. A $100 \mathrm{~mm} \times 28 \mathrm{~mm}$ area around the hole was laser scanned at intervals of $0.3 \mathrm{~mm}$ to generate ultrasonic waves, and the propagation waveforms were detected by an angle beam transducer $\left(90^{\circ}\right.$ incidence, nominal frequency: $2.25 \mathrm{MHz}$ ) mounted $100 \mathrm{~mm}$ from the right end. Figure 8 shows the visualized images for each $4 \mu$ s interval of propagation time. When ultrasonic waves impinged on the drill hole with a diameter of $1 \mathrm{~mm}$, the waves scattered from the hole (see images for $4 \mu \mathrm{s}$ and $12 \mu \mathrm{s}$ ) and then spread radially (see images for $8 \mu \mathrm{s}$ and $16 \mu \mathrm{s}$ ). The 
spiral-form waves shown in Fig. 8 are the waves propagating within the round tube.

Finally, we introduce an example of the visualization of ultrasonic waves propagating on carbon fiber reinforced plastics (CFRP), which has been difficult with the conventional laser probe scanning method because of poor detection sensitivity. Figure 9 shows a photograph of the laboratory apparatus used for visualizing ultrasonic waves propagating on CFRP. With an AE sensor mounted on the back of a flat CFRP plate with dimensions of $290 \times 140 \times 2 \mathrm{~mm}\left(0 / 90_{4 \mathrm{~S}}\right)$, the front side was laser scanned, and the propagated waves were detected. To examine how a flaw changes the propagation of ultrasonic waves, images of the ultrasonic propagation after delaminating the specimen by a hammer strike were also measured and compared with those of the intact specimen. Figure 10 compares the images for the two cases. The disturbance in the waveforms that occurred after the ultrasonic waves passed the damaged portion is visualized. Furthermore, anisotropy in the $\mathrm{S}_{0}$ wave velocity and isotropy in the $\mathrm{A}_{0}$ wave velocity ${ }^{(12)}$ are clearly observed. A PZT sensor with a sensitivity 10 to 100 times higher than that of a detection laser probe was used as the reception sensor in this measurement. As shown by the waveform detected at point $\mathrm{E}$ and its frequency spectrum, shown at the top of Fig. 10, a sensor with a resonant frequency of $500 \mathrm{kHz}$ was used for detecting ultrasonic waves in this experiment due to its high sensitivity. As a result, the dispersion of velocity depending on frequency was rarely observed in the propagated Lamb waves.

\section{Conclusion}

We have proposed a method wherein thermally excited ultrasonic waves are generated by scanning pulsed-laser beams on the specimen and the propagation of the ultrasonic waves is visualized based on the train of waveforms detected by a reception sensor mounted at a fixed point. The conventional method, in which ultrasonic waves are visualized by 
scanning the detection laser probe, is only applicable to objects that have smooth surfaces and has very poor operability and work efficiency because of the need for sophisticated optical adjustment, which means that the conventional method can be used to study ultrasonic waves only in the laboratory. In contrast, because of its capacity for efficient scanning, the proposed method enables the visualization of ultrasound propagating on a 3-D object of arbitrary shape, and is considered to be appropriate for flaw inspection in the field. In the near future, we will develop a measurement systems that enables further high-speed and high-accuracy visualizations and will examine their possible use in flaw inspection of piping and similar applications.

\section{References}

(1) Dilhan K. L., Don-Liyanage and David C. Emmony, Schlieren imaging of laser-generated ultrasound, Appl. Phys. Lett., 79-20, pp.3356-3357(2001)

(2) Breazeale M. A., From monochromatic light diffraction to color schlieren photography, $J$. Optics A, 3-4, pp.1-7(2001)

(3) Declercq N. F., et. al., Study of the scattering of leaky Rayleigh waves at the extremity of a fluid-loaded thik plate, J. Appl. Phys., 96-10, pp.5838-5840(2004)

(4) Nam Y. H. and Lee S.S., A quantitative evaluation of elastic wave in solid by stroboscopic photoelasticity, J. Sound and Vibration, 259-5, pp.1199-1207(2003)

(5) Mihara T., Visualization of propagation of ultrasounds by photo-elastic imaging process (in Japanese), Trans. of the Visualization Soc. of Jpn., 118-70, pp.181-186(1988)

(6) Ghorayeb, S. R., Xue, T. and Lord, W., Ultrasonic wave propagation in a tooth phantom, Review of Progress in Quantitative Nondestructive Evaluation, 7B, pp.1721-1728 (1998)

(7) Hayashi T. and Kawashima K., Analysis of flexursl mode focusing by a semianalytical finite element method, J. Acoust. Soc. Am., 113-3, pp.1241-1248(2003)

(8) Yamawaki H. and Saito T., Numerical calculation of ultrasonic propagation with anisotropy, NDT \& E Int. J, 33, pp.489-497(2000)

(9) Schechter R.S., et. al., Use of transient wave propagation code for 3D simulation of $\mathrm{cw}$ radiated transducer fields, Ultrasonics, 37, pp.89-94(1999)

(10) Takatsubo J., Imade M., Fan Q. and YamamotoS., Visualization of elastic waves by digital laser ultrasonics (in Japanese), Trans. of the Jpn. Soc. Of Mech. Engineers, Series C, 65-639, pp.4299-4304(1999)

(11) Takatsubo J. and Imade M., Visualization of ultrasonic waves propagating around artificial defects (in Japanese), Trans. of the Visualization Soc. of Jpn., 22-3, pp.23-27(2002)

(12) Toyama N., et. al, Two-dimensional AE source location with two sensors in thin CFRP plates, J. Mater. Sci. Lett., 20, pp.1823-1825(2001) 